\title{
Treatment of Walled-off Necrosis - Outcomes are Best When Endoscopy and Percutaneous Interventions are Complimentary, Not Exclusive
}

\author{
Ji Young Bang and Shyam Varadarajulu \\ Digestive Health Institute, Orlando Health, Orlando, Florida, USA
}

See "RWON Study: The Real-World Walled-off Necrosis Study" by Ankush Pawar, Ujjwal Sonika, Manish Kumar, et al., on page 909-915.

\begin{abstract}
Necrotizing pancreatitis occurs in one-fifth of patients with severe acute pancreatitis and is associated with high mortality, particularly when secondary infection results in sepsis and organ failure. ${ }^{1,2}$ Considering open necrosectomy is associated with high rates of post-surgical complications and mortality, alternative techniques that incorporate a step-up approach of endoscopic transluminal drainage or percutaneous catheter placement with subsequent endoscopic or minimally invasive debridement have been advocated. These new treatment approaches have significantly reduced the rates of disease and procedure-related morbidity and mortality. ${ }^{3}$

The majority of data on walled-off necrotic collections are derived from the context of clinical investigations or collected retrospectively. In the study published in this issue of Clinical Endoscopy, which was conducted in New Delhi, the clinical data were collected prospectively over 4 years in 264 patients. ${ }^{4}$ Approximately every one-third cohort of patients was managed conservatively, radiologically, or endoscopically, and followed up for up to 3 months. The data support the conclusion that endoscopic drainage, compared to percutaneous meth-
\end{abstract}

\footnotetext{
Received: October 13, 2021 Revised: October 20, 2021

Accepted: October 20, 2021

Correspondence: Shyam Varadarajulu

Digestive Health Institute, Orlando Health, 1335 Sligh Blvd, Orlando, FL 32806, USA

Tel: +1-321-841-9022, Fax: +1-321-843-6295, E-mail: svaradarajulu@yahoo.com ORCID: https://orcid.org/0000-0002-0047-0016
}

(c) This is an Open Access article distributed under the terms of the Creative Commons Attribution Non-Commercial License (http://creativecommons.org/ licenses/by-nc/3.0) which permits unrestricted non-commercial use, distribution, and reproduction in any medium, provided the original work is properly cited. ods, was associated with lower mortality, fewer complications, and shorter hospitalization. The conclusions are expected because unlike the endoscopic approach, in which patients after transluminal drainage underwent endoscopic necrosectomy for non-resolution of symptoms at 72 hours, those treated by radiological drain placement did not receive standardized care. They were managed on an as-needed basis with upsizing of drains. Although commonly practiced, this method of treatment is not the current standard of care. Percutaneous drain placement is only the first step in a step-up protocol where, based on treatment response, patients subsequently undergo video-assisted retroperitoneal debridement or percutaneous necrosectomy. Naturally, the upsizing of drains without addressing the underlying solid debris results in the persistence of symptoms, prolonged hospitalization, frequent readmissions, and higher mortality. Surprisingly, only $4 \%$ of 264 patients with necrotic collections underwent treatment integrating radiological drainage and endoscopic stent placement. In our experience, a significant proportion of patients require multimodality management with endoscopic stent or radiological drain placement as the only first step in the treatment algorithm. Additionally, given the short follow-up duration of 3 months, the consequences of a disconnected pancreatic duct in either cohort that leads to recurrence of fluid collection and/or development of fistulas in the long term could not be addressed.

A central theme in the management of pancreatic fluid collection is the multidisciplinary approach. Endoscopic, radiological, and surgical disciplines must coordinate in routine clinical practice. The treatment protocol must integrate these 
three approaches with a focus on both near-term and longterm consequences, such as symptom and fluid collection recurrence. In addition, treatment algorithms must be standardized for reinterventions, and the pancreatic duct status must be considered, as they have long-term prognostic implications. ${ }^{5}$ Such an approach will yield optimal outcomes for all patients and not just those managed by endoscopic interventions.

Conflicts of Interest

Ji Young Bang is consultant for Boston Scientific, Olympus, Fujifilm. Shyam Varadarajulu is consultant for Boston Scientific, Olympus, Medtronic

Funding

None.

Author Contributions

Writing - original draft: Shyam Varadarajulu

Writing - review \& editing: Ji Young Bang
ORCID

Shyam Varadarajulu:

https://orcid.org/0000-0002-0047-0016

Ji Young Bang:

https://orcid.org/0000-0001-9351-3267

\section{REFERENCES}

1. Tenner S, Baillie J, DeWitt J, Vege SS, American College of Gastroenterology. American college of gastroenterology guideline: management of acute pancreatitis. Am J Gastroenterol 2013;108:1400-1415;1416.

2. Whitcomb DC. Clinical practice. Acute pancreatitis. N Engl J Med 2006;354:2142-2150.

3. van Santvoort HC, Besselink MG, Bakker OJ, et al. A step-up approach or open necrosectomy for necrotizing pancreatitis. N Engl J Med 2010;362:1491-1502.

4. Pawar A, Sonika U, Kumar M, Saluja S, Srivastava S. RWON study: the real-world walled-off necrosis study. Clin Endosc 2021;54:909-915.

5. Bang JY, Wilcox CM, Arnoletti JP, Peter S, Christein J, Navaneethan U, et al. Validation of the Orlando Protocol for endoscopic management of pancreatic fluid collections in the era of lumen-apposing metal stents. Dig Endosc 2021 Jul 31 [Epub]. https://doi.org/10.1111/den.14099. 